\title{
Variations
}

Variations

Revue internationale de théorie critique

$9 / 10 \mid 2007$

Les frontières de la politique

\section{Quartett de Heiner Müller : le désir et le vide}

\section{Laure Couillaud}

\section{(2) OpenEdition}

\section{Journals}

Édition électronique

URL : http://journals.openedition.org/variations/478

DOI : 10.4000/variations.478

ISSN : 1968-3960

\section{Éditeur}

Les amis de Variations

\section{Édition imprimée}

Date de publication : 1 juin 2007

Pagination : 107-118

\section{Référence électronique}

Laure Couillaud, «Quartett de Heiner Müller : le désir et le vide», Variations [En ligne], 9/10 | 2007, mis en ligne le 01 décembre 2012, consulté le 19 avril 2019. URL : http://journals.openedition.org/ variations/478; DOI : 10.4000/variations.478

Ce document a été généré automatiquement le 19 avril 2019.

Les ami•e•s de Variations 


\title{
Quartett de Heiner Müller : le désir et le vide
}

\author{
Laure Couillaud
}

1 Voici les dernières lignes de la pièce Mauser, écrite en 1970 par l'auteur dramatique Heiner Müller (1929-1995). Pièce sur le thème de la révolution, réflexions sur l'homme face à ce cas extrême : être l'ami ou bien l'ennemi de la révolution, c'est-à-dire ne pas avoir d'autre choix que de tuer ou d'être tué, de fusiller ou d'être fusillé. Jean-Pierre Morel, auteur d'un essai sur Müller, a distingué trois sortes d'expériences, qu'il nomme aussi «strates», dans l'œuvre: l'expérience politique, l'expérience amoureuse, et l'expérience esthétique. Mauser appartient à la strate politique et sociale ${ }^{2}$. Quant à elle, la pièce Quartett ${ }^{3}$, écrite en 1980, appartient à la strate amoureuse. Elle met en scène les deux protagonistes du roman de Laclos Les Liaisons dangereuses (1782), Valmont et Merteuil. Mauser et Quartett sont par leur thématique des pièces bien différentes, et pourtant Müller lui-même a reconnu dans ces deux pièces la même structure, la même dramaturgie. L'écriture de Quartett était un projet qui existait depuis les années cinquante, et c'est seulement après avoir vu une mise de scène de Mauser par Christoph Nel à Cologne en 1980, qu'il a pu l'écrire :

Le dramaturge de Christoph Nel était Urs Troller, tous deux étaient issus de bonnes maisons bourgeoises et étaient politiquement exempts de tout péché. Ils pensaient donc pouvoir représenter le tout simplement en étudiant une relation hommefemme. C'était la seule relation de pouvoir qu'ils connaissaient d'après leur propre domaine d'expérience et de vie. Mauser a donc été joué par un homme et une femme et, à chaque coup de feu, l'homme écrasait une tarte à la crème entre les cuisses de la femme. Cela a l'air assez bête, mais ce n'était pas si élémentaire que ça. Quand ensuite j'ai écrit Quartett, je savais qu'avec Mauser, c'était Quartett qu'ils avaient mis en scène ${ }^{4}$.

2 Un homme souillant l'entrecuisse d'une femme avec de la tarte à la crème à chaque fois qu'un homme est fusillé, ce serait donc cela Quartett, le bruit de la mort qui déclenche un geste obscène, qui salit un espace sacré, source de vie, autant qu'un espace obscur, source de jouissance. L'image scénique (répétée) et donc visible d'un sexe souillé, comme réplique à l'image invisible d'un corps blessé par balle, d'un corps qui tombe. C'est cela, 
Quartett : un espace de désacralisation, qui pose la question du désir, et un espace de chute dans la mort, qui pose la question du vide.

Müller croit à la nécessité de l'ouverture - réouverture du dialogue avec les spectres, il est l'auteur d'un «théâtre de la résurrection »:

L'une des fonctions du drame est la conjuration des morts - le dialogue avec les morts ne doit pas s'interrompre avant qu'ils n'aient rendu la part d'avenir qui est enterrée en eux. [...] Si nous avons un théâtre, ce sera un théâtre de la résurrection ${ }^{5}$

...

4 Müller a réécrit de nombreux mythes, a convoqué de nombreux héros du passé dans ses textes: Prométhée, Médée, Hamlet, Héraclès, etc. La dimension mythique du couple Valmont/Merteuil est sans aucun doute à l'origine de ce travail d'écriture-réécriture des Liaisons qu'est Quartett. Qui sont Valmont et Merteuil dans le roman de Laclos? Des libertins oisifs, qui excellent dans l'art de la perversion et de la persuasion, des êtres hors du commun qui se dominent eux-mêmes et qui dominent les autres, des comédiens dont le terrain de jeu privilégié est l'érotisme. Spectateurs d'eux-mêmes, chacun est pour l'autre un public de choix, chacun est pour l'autre à la fois le miroir et l'adversaire. Ce couple fascinant, modèle d'un dépassement mythique de soi, Müller l'a déterré en cassant les jouets de Laclos, comme il le dit lui-même, en détruisant les clichés et les refoulements. Cet acte de réécriture va autopsier, disséquer ces héros de la démesure, ces héros de la guerre des sexes. Et par là faire jaillir une vérité, peut-être universelle, celle du pouvoir et du désir, celle du squelette de la relation homme-femme.

5 Dans Quartett, Valmont et Merteuil ressuscitent le temps d'un dernier combat, dans une "période» donnée en indication scénique, à la fois concrète et utopique: Un salon d'avant la Révolution française - Un bunker d'après la troisième guerre mondiale. En équilibre entre la mémoire du passé et la mort, ces deux "naufragés de la littérature » conscients de leur naufrage (re) jouent leur guerre une dernière fois, avec pour seule arme le langage. Le texte est une succession de phrases explosives, aussi spirituelles qu'obscènes. Les mots, les images, les actes décrits dans Quartett abondent d'animalité, de bestialité, de férocité, Valmont le dit lui-même :

Quel ennui que la bestialité de notre conversation. Chaque mot ouvre une blessure, chaque sourire dévoile une canine. Nous devrions faire jouer nos rôles pas des tigres. Encore une morsure, encore un coup de griffe? L'art dramatique des bêtes féroces.

Que doit-on entendre par «dramatique»? Théâtral ? Catastrophique? Pathétique ? Le texte joue sur les frontières du drame et multiplie les interprétations et donc les interrogations. Le texte joue à peindre plusieurs couches dans le cadre de l'enfermement, joue sur la forme et le contenu du désir érotique, et pour finir déchire le tableau délimité par l'enfermement, cet enfermement qui condamne Valmont et Merteuil à l'expérience intérieure. Valmont et Merteuil sont-ils des animaux, des comédiens ou des hommes ? La mort, écrit Georges Bataille, rejette l'homme dans l'animalité ${ }^{6}$. Valmont et Merteuil sont dans la conscience et le temps de leur mort, ce qui explique la bestialité de leur conversation. Ils connaissent la vulgarité inévitable de la mort: l'homme meurt comme un chien. Ils jouent avec le « dégoût et la séduction fiévreuse » qui s'exaspèrent dans la mort. « Dans le halo de la mort, et là seulement, le moi fonde son empire; là se fait jour la pureté d'une exigence sans espoir ; là se réalise l'espoir du moi-qui-meurt ${ }^{7}$ ", écrit encore Bataille. Les corps de Valmont et Merteuil désincarnés par Müller subissent consciemment l'expérience intérieure de la mort. Si les vivants défient l'horreur du vide, l'horreur de n'être plus rien, Valmont et Merteuil avec ironie remplissent ce vide, sans 
avoir le vertige, impassiblement, insensiblement. Ils vivent leur mort et contemplent leur chute. Ils n'ont plus le désir éperdu d'être eux-mêmes. La preuve : ils jouent à être autre chose qu'eux-mêmes, ils jouent à être l'autre, ils jouent à travers et pour l'autre. Ils jouent à redétruire les jouets d'autrefois, à savoir deux victimes «idéales » du sexe masculin, deux victimes «idéales » de Valmont, deux modèles poussés à la caricature du sujet féminin : Cécile de Volanges, jeune vierge à dépuceler fraîchement sortie du couvent, et Madame de Tourvel, femme mariée et pieuse, morte d'avoir aimé Valmont jusqu'à l'infidélité. Leur jeu est superficiellement la réécriture de deux mises à mort, deux anéantissements, l'un par le sexe, l'autre par la mort. Valmont et Merteuil sont, dans Quartett, détachés de toute contrainte, hors d'atteinte, loin de la grande scène " sociale " sur laquelle ils ont brillé, grâce au langage, et parce que, comme deux anciens combattants, ils n'ont plus rien à perdre, vont créer un espace de jeu. Le langage, dans Quartett, semble surgir du néant, et apparaît comme une zone de survivance, comme une spatialité non nihilisable, comme une force active qui naît du silence. Le théâtre peut commencer.

7 Ce langage, source et révélateur ici de tous les excès, semble être leur ultime source de jouissance. Car, comme dans le temps du roman de Laclos, Valmont et Merteuil ne jouiront plus ensemble. Merteuil est seule en scène au début de la pièce :

Valmont. Je la croyais éteinte votre passion pour moi. D'où vient ce soudain retour de flamme. Et d'une passion si juvénile. Trop tard bien sûr. Vous n'enflammerez plus mon cœur. Pas une seconde fois. Jamais plus. [...] Quand je ferme les yeux, vous êtes beau, Valmont. Ou bossu, si je veux. [...] Ne retirez pas votre délicieuse proposition, Monsieur. J'achète de toute façon. [...] Ne vous pressez pas, Valmont. Comme cela c'est bien. Oui oui oui oui. C'était bien joué, non. Qu'importe la jouissance de mon corps...

8 Il y a, dès l'ouverture du texte, dans la solitude de Merteuil, un sentiment implacable de «trop tard», et le fantasme, qu'elle sait pathétique, du retour de Valmont ${ }^{8}$. Merteuil simule un orgasme, se moque de la jouissance et se joue de Valmont, mais ne semble jouer que pour elle-même. Pourquoi? Peut-être parce qu'elle est piégée par la solitude, enfermée avec ses souvenirs et l'image de l'être aimé. Elle affronte et mesure, non sans ironie, le gâchis de leur histoire. À la fin du monologue, elle dit : "Je suis tout à fait froide, Valmont. Ma vie Ma mort Mon bien-aimé.» Entre jouissance impossible des deux corps et amour éternel, elle se débat avec elle-même, et tente peut-être de s'arracher à l'imaginaire amoureux. À ce moment-là, Valmont apparaît, il entre en scène. Entrée de L'autre, présence de l'absent, peut-être même qu'il faut aller plus loin : résurrection de l'autre ; le dialogue, interrompu par/dans le passé peut reprendre.

Je tente de m'arracher à l'imaginaire amoureux, mais l'imaginaire brûle pardessous, comme de la tourbe mal éteinte; il s'embrase de nouveau; ce qui était renoncé resurgit ; de la tombe mal fermée surgit un long cri'.

Ce passage écrit par Roland Barthes dans les Fragments d'un discours amoureux sur le thème de l'exil est bien le point de commencement, et le fondement même de la pièce. Quartett est bien « un long cri » qui resurgit d'une tombe mal fermée, cette tombe n'est autre que la tombe de la passion amoureuse. Le dialogue de Valmont et Merteuil semble être en quelque sorte l'extension du deuil amoureux. Il faut entendre par extension la libération du deuil amoureux refoulé. Dans Quartett, Müller ne se contente pas de libérer ce refoulement, il le détruit. Destruction totale, qui ne pouvait pas avoir lieu sans la disparition, la mort réelle de Valmont. L'entrée de Valmont au début de la pièce est la fois la renaissance du souvenir et le premier pas vers sa propre destruction. Dans le deuil réel, 
écrit encore Roland Barthes, c'est l'épreuve de réalité qui me montre que l'objet aimé a cessé d'exister. Dans le deuil amoureux, l'objet n'est ni mort, ni éloigné. C'est moi qui décide que son image doit mourir ${ }^{10}$. C'est ce qui se passe dans Quartett: Merteuil ellemême ou son imaginaire, ambiguïté sublime de la pièce, va mettre à mort l'image de Valmont. Mais grâce au jeu de masques, grâce au "théâtre dans le théâtre » cette mise à mort va se concrétiser sur scène. L'épreuve de l'imaginaire, le langage de l'imaginaire devient grâce au théâtre l'épreuve de réalité. Merteuil va tuer Valmont, objet de son amour. Les derniers mots de Merteuil mêlés au dernier souffle de Valmont sont sans appel: "À présent nous sommes seuls, cancer mon amour». Le cercle de la solitude se referme: Merteuil est définitivement seule au monde. Valmont a cessé d'exister, disparition physique de l'objet vénéré. Le théâtre est le lieu exceptionnel où le langage de l'imaginaire et la réalité se retrouvent face à face. Le théâtre qui s'inscrit nécessairement, toujours dans un "ici et maintenant", est le seul espace de représentation où l'extrémisation du deuil amoureux pouvait avoir lieu : mort réelle et mort de l'image de l'objet vénéré. Cette extrémisation porte en elle un processus de destruction implacable. Le jeu apparaît dans Quartett comme l'expérience-limite du couple. Ce jeu a "produit » une vérité effrayante et pose la question de l'après-destruction. Dans ses Écrits sur le théâtre, Roland Barthes distingue dans l'œuvre de Brecht deux discours, puis un troisième. C'est le premier, dont relèvent les premières pièces de Brecht, qui nous intéresse ici, le discours apocalyptique (anarchisant) : «Il s'agit de dire et de produire la destruction, sans chercher à savoir ce qui vient après...11 ». Dans Quartett, la solitude de Merteuil et la mort de Valmont signifient bien l'absence d'après. Le jeu est fini, le silence de la mort et le silence de la solitude résonnent, mais on ne sait pas ce qui vient après, rien n'existe après, si ce n'est le vide, puisque Dieu n'existe pas. Cela donne lieu à une interprétation nihiliste de la pièce. À la fin du texte, Merteuil donne un verre de vin empoisonné à Valmont. Si ce dernier consent à mourir en acceptant le verre ("Vous n'avez pas besoin de me dire, Marquise, que le vin était empoisonné»), il n'en est pas moins vrai que Merteuil tue Valmont en toute impunité. Mais en tuant Valmont, n'est-elle pas condamnée à subir le vide, n'estelle pas elle-même condamnée à disparaître («Cancer mon amour », dit-elle). Nous pouvons lire dans le Gai savoir :
Ne serions-nous pas [...] tombés dans le soupçon d'un contraste, le contraste entre ce monde où, jusqu'à présent, nous avions le sentiment d'être chez nous avec nos vénérations - ces vénérations à cause desquelles nous supportions peut-être de vivre - et un monde qui n'est autre que nous-mêmes: un soupçon implacable, foncier et radical à l'égard de nous-mêmes, qui s'empare toujours davantage de nous autres européens, nous tient toujours plus dangereusement en sa puissance, et pourrait facilement placer les générations futures devant cette terrible alternative : «Supprimez ou vos vénérations, ou bien vous-mêmes!» Le dernier cas aboutirait au nihilisme, mais le premier cas n'aboutirait-il pas aussi au nihilisme? C'est là notre point d'interrogation $!^{12}$

Même si les mots de Nietzsche nous éloignent de la thématique amoureuse, Merteuil n'est-elle pas dès le début de la pièce comme ces "générations futures " placée devant cette " terrible alternative ", face à deux mondes : sa solitude et son désir ? Cette mise en parallèle, interprétation du vide que laisse " après coup » le jeu et le texte, pourrait être aussi l'interprétation de cette période donnée en indication: "un salon d'avant la Révolution française - Un bunker d'après la troisième guerre mondiale ». Un salon, comme espace de jeu privilégié pour libertins oisifs, et un bâtiment de guerre hermétiquement fermé, roc au milieu d'un paysage dévasté par la guerre, le contraste est saisissant et configure un espace-temps introuvable en équilibre entre le passé et l'avenir, 
entre un événement historique qui a existé et un autre qui pourrait exister. Ce contraste est peut-être celui dont parle Nietzsche. Le salon, comme mythe daté de la cohabitation de soi et de l'objet vénéré, qui n'est autre que la problématique du désir. Le bunker, comme la part d'avenir du mythe de la cohabitation et comme la part d'avenir immanente au désir : la négation de soi (la mort) ou la négation de l'autre (la solitude).

11 La solitude de Merteuil est un vide plein, la mort de Valmont est un vide définitif. Quand Georges Bataille parle du manque, il parle d'un vide «devant lequel l'être est un plein, menacé de perdre la plénitude, désirant et craignant également de la perdre ${ }^{13}$.» $\mathrm{Ce}$ manque, entre menace de perdition et désir de perdition, met à l'épreuve l'être et révèle son excès d'être. L'affirmation du vide est l'affirmation d'une menace, celle du désir, qui trouve son fondement dans le manque à être. Le jeu à être l'autre dans Quartett, extrémise le désir et l'affirme dans cette terrible alternative: Se perdre soi ou perdre l'autre. Quartett, pour cela, est l'écho, la vision, sur fond d'érotisme, de ce point d'interrogation que pose Nietzsche. La pièce est profondément nihiliste, mais semble l'être par nécessité : pour affronter les frontières du corps et de l'esprit humain, il faut créer l'expérience limite du désir, et par là affirmer et libérer l'objet désir à travers la négation des sujets et des corps, à travers l'impossible réconciliation, un manque jamais comblé et son éclatement par le vide.

L'exploration des limites au théâtre s'accomplit aujourd'hui sans les certitudes métaphysiques et politiques ni les normes esthétiques qui sous-tendaient la poétique antique, garantissant alors et sécurisant en quelque sorte le but cathartique de l'art. La voie négative doit être ouverte sans garantie aucune, sans espérance affichée...14

12 Si l'écriture est l'espace de la destruction et le théâtre son espace privilégié de communication, l'exploration de la voie négative devient alors une voie affirmative grâce à la représentation. Le théâtre est le lieu où l'affirmation de la négation et la libération de cette même négation peuvent avoir lieu dans un même temps, dans un espace hors temps. Dans Quartett, cette affirmation/libération ne pouvait exister sans l'anéantissement du sujet masculin. Par le langage d'abord. En désacralisant la jouissance et la puissance phallique. De Merteuil à Valmont dans le texte :

Qu'avez-vous appris si ce n'est à manœuvrer votre queue dans un trou en tous points semblable à celui dont vous êtes issu, avec toujours le même résultat, plus ou moins divertissant, et toujours dans l'illusion que l'applaudissement des muqueuses d'autrui va à votre seule personne, que les cris de jouissance sont adressés à vous, alors que vous n'êtes que le véhicule inanimé de la jouissance qui vous utilise, indifférent et tout à fait interchangeable, bouffon dérisoire dans sa création. Vous le savez, pour une femme tout homme est un homme qui fait défaut.

Puis par l'acte criminel de Merteuil portant le masque de Valmont :

Vous permettez, je prends mon lorgnon pour mieux voir le spectacle, votre dernier, ma reine, avec crainte et pitié. J'ai fait installer des miroirs pour que vous puissiez mourir au pluriel. Et faites-moi la joie de recevoir de mes mains ce verre de vin, votre dernier.

Que signifie mourir au pluriel ? La mort de soi et la mort de l'autre, la mort du bourreau portant le masque de sa victime, la mort de la victime portant le masque du bourreau. Le jeu de masques, étant à la fois la mise en scène de soi-même et la mise en scène de l'autre, permet la coexistence du je et de l'autre dans un seul corps. Maurice Blanchot, dans son commentaire de La Maladie de la mort de Marguerite Duras, écrit au sujet de la fin du texte : cette fin (la disparition de la femme et donc la perte de l'amour) n'est pas «l'échec de l'amour dans un cas singulier, mais l'accomplissement de tout amour véritable qui 
serait de se réaliser sur le seul mode de la perte, c'est-à-dire de se réaliser en perdant non pas ce qui vous a appartenu mais ce que l'on n'a jamais eu, car le «je » et «l'autre » ne vivent pas dans le même temps, ne sont jamais ensemble (en synchronie), ne seraient donc jamais contemporains, mais séparés (même unis) par un " pas encore » qui va de pair avec un « déjà plus » ${ }^{15}$. » La mort de Valmont, perte synchronique de soi et de l'autre, symbolise bien cette séparation irréductible, celle mise en jeu dans le désir, celle des deux chairs. Mais la mort de Valmont n'est-elle pas aussi la réconciliation de l'être avec luimême, de l'être et de sa part incontrôlable? Le sujet masculin, écrit Günther Heeg ${ }^{16}$ dans une analyse des figures müllériennes, "ne peut pas vivre avec en lui-même l'« autre » incontrôlable, car cela lui rappelle les limites de son pouvoir, sa faiblesse et sa finitude. ${ }^{17}$ " Le sujet masculin doit pour vivre nier cette part de lui-même, autant incontrôlable qu'inavouable, mais cette part le hante. Pour Valmont, cette part n'est autre que son désir sans fin pour le sexe féminin. À la fin de la pièce, Valmont disparaît en femme, il devient ce qu'il voulait à tout prix posséder. À force de conquérir le sexe des femmes, à force de vouloir le posséder, de faire de ce sexe un miroir réfléchissant l'image de sa puissance, ou plutôt de sa volonté de puissance, il meurt dépourvu de son sexe, il ne sera plus jamais le conquérant d'autrefois, il consent à mourir dans la peau d'une femme, celle de son ultime "victime », victime suicidée, Tourvel. «Je crois que je pourrais m'habituer à être une femme marquise » lance-t-il. "Le sacrifice de la dame ", comme le dit Merteuil, est aussi l'agonie de Valmont. À la fin de la pièce, Valmont "victime de Merteuil » incarne l'objet de son obsession : désir de jouir de Tourvel, qui incarne à la fois la résistance et l'amour pur. Cette mise à mort, entre acte criminel et acte suicidaire, n'aurait pas pu exister, si Merteuil grâce au jeu ne possédait pas elle-même à ce moment-là les propriétés mâles du séducteur. Valmont et Merteuil, dans une quête de jouissance absolue et d'amour ne cherchent-il désespérément - ce qui n'exclut pas l'humour - «la partie dont il a été dépourvu, et cette recherche est le véritable moteur de l'action comme de l'amour ${ }^{18}$.» Chacun homme/femme, chacun en alternance chasseur et proie, chacun traversé par des fragments d'identité du sexe opposé, exprime un désir de possession de l'autre. Mort d'une putain, dit Merteuil au sujet de Valmont. Ces mots « orduriers » ne sont-ils pas ceux d'un homme, peut-être ceux d'un homme frustré dépourvu de son agressivité virile, une femme. On note ici un fait important : Valmont ne jouera jamais le «rôle» de Merteuil, fait qui aiguise l'argumentation qui concerne l'anéantissement du sujet masculin.

Mort d'une putain: Valmont a conscience de sa mort, et les mots de Merteuil mettent à nu sa déchéance, sa déchéance d'homme. On perçoit dans les mots de Merteuil cette volonté de traiter l'autre en objet, volonté de destituer Valmont de sa personnalité de prédateur, qui n'est autre que son sexe, sa part incontrôlable... Un passage de l'Érotisme de Georges Bataille, au sujet de la basse prostitution, qu'il analyse comme le dernier degré de l'affaissement, pourrait expliquer la fin de la pièce, et les mots violents de Merteuil à l'égard de Valmont:

Celui qui, d'un mourant, dit qu'« il va crever » envisage la mort d'un homme comme celle d'un chien, mais il mesure la déchéance, l'affaissement, qu'opère le langage ordurier qu'il emploie. Les mots grossiers, les organes, les produits ou les actes sexuels introduisent le même affaissement. Ces mots sont interdits, il est généralement interdit de nommer ces organes. Les nommer d'une manière éhontée fait passer de la transgression à l'indifférence qui met sur un même pied le profane et le plus sacré. La prostituée de bas étage est au dernier endroit de l'affaissement. Elle pourrait n'être pas moins indifférente aux interdits que l'animal, mais impuissante à parvenir à la parfaite indifférence, elle sait des interdits que les autres observent: et non seulement elle est déchue, mais la possibilité lui est 
donnée de connaître sa déchéance. Elle se sait humaine. Même sans honte, elle peut avoir conscience de vivre comme les porcs. ${ }^{19}$

Si Valmont est réduit, à la fin de la pièce, à être une femme, réduite elle-même à la prostitution, n'y a-t-il pas dans cette déchéance poussée à l'extrême un espoir de jouissance, au-delà de la mort, au-delà de la non-vie? «Je voudrais pouvoir assister à votre mort comme j'assiste maintenant à la mienne. D'ailleurs je me plais encore à moi-même. La masturbation continue avec les vers ", dit Valmont avant de mourir. La jouissance se mêle à la mort. Dans la décomposition, la putréfaction de la chair, la jouissance se prolonge : affirmation de la vie dans l'horreur de la mort. Valmont est aussi l'auteur de ces mots : « Seule la jouissance peut enlever à l'amour son bandeau et lui faire voir à travers le voile de la peau la nudité de la chair, nourriture indifférente des tombeaux. » Quartett est un texte qui détruit la jouissance dans le temps du texte, mais qui la reconstruit dans la conscience et le temps de la mort. Merteuil, plus haut dans le texte, dit ceci :

Quelque chose vit entre l'homme et la bête. Que j'espère ne pas avoir à rencontrer, ni dans cette vie, ni dans une autre, à supposer qu'il y en ait une autre. À la seule pensée de son odeur, je sue de tous mes pores. Mes miroirs exsudent son sang. Cela ne trouble pas mon image, je ris du tourment des autres comme tout animal doué de raison. Mais il m'arrive de rêver qu'il surgit de mes miroirs sur ses pieds de fumier et sans visages, mais je vois ses mains avec précision, griffes et sabots, quand il m'arrache la soie des cuisses et se jette sur moi comme la terre sur un cercueil, et peut-être sa violence est-elle la clef qui ouvre mon cœur.

Ces mots violents et profondément morbides, expressions d'un fantasme, métaphore de la passion amoureuse, montrent le lien intime qui existe entre l'érotisme et la mort, entre le désir et la haine. Comme si le véritable amour naissait d'un viol. Qu'est-ce qui fait de nous des êtres humains? Quelque chose vit entre l'homme et la bête. La violence du désir peut faire surgir des actes inhumains. Dans l'espace du rêve, là où l'idée de transgression et d'interdit n'a pas lieu d'être, là où le désir s'exacerbe sans limites aucune, Merteuil révèle l'impossible isolement de l'angoisse de mort liée à la chair « objet de désir ou source de jouissance "... La pulsion érotique, quasi animale qui déchire la nudité de la chair, décrite et fantasmée par Merteuil, étant comme la terre jetée sur un cercueil. Du début à la fin du texte, les personnages sont obsédés par la mort, peut-être parce qu'ils sont obsédés par la jouissance, mais qu'ils en connaissent le prix: l'angoisse de la mort, qui est aussi l'angoisse du temps qui passe, l'angoisse de la nuit des corps.

"Mettre à plat» le conflit homme-femme, mettre en jeu une opposition, une rivalité originelle, c'est mettre en jeu le théâtre d'une tentative de réconciliation, et affronter l'impossibilité de cette réconciliation. Dans Quartett, la guerre des sexes est le miroir des relations de pouvoir inhérentes au désir et à l'amour. Si le désir, comme le dit Matthias Langhoff ${ }^{20}$, détermine les règles du jeu, la limite du jeu est bien, comme nous l'avons vu, le vide. Ce vide est dans un même temps, le temps de la finitude de l'être et le temps de la certitude d'être, pour soi-même et pour l'autre. Ce vide, temps de l'isolement de l'être (disparition de l'autre) et temps de la réconciliation de l'être à travers la mort (disparition de soi, décomposition de la chair), semble être un temps inhumain, suspendu, sans passé ni avenir, qui éclaire notre présent, temps humain toujours illisible, indéfinissable, concept déterminé par le passé et l'avenir. Cette affirmation d'inhumanité par le vide n'est-elle pas porteuse d'éternel, grâce au présent de la représentation, et grâce à l'absence d'après, résultat d'un combat à mort? Kierkegaard écrit dans Le Concept d'angoisse que l'éternel est [au contraire] le présent: Pour la pensée, l'éternel est le présent conçu comme la suppression de succession (le temps étant la succession qui 
passe), pour la représentation, c'est un progrès sur place, car il est pour elle un présent infiniment plein. On ne retrouve donc pas dans l'éternel la distinction de passé et d'avenir, parce que le présent est posé comme étant la suppression de la succession ${ }^{21}$. Si le présent est un concept infiniment vide, il est aussi l'éternel. Le théâtre " art au présent ", art de l'actualisation, est un espace de représentation où la mort et l'éternité peuvent coexister. Cet espace est, dans Quartett, l'espace privilégié du ressouvenir. Ce dernier est double dans la pièce de Müller, parce qu'il s'agit d'un théâtre de la résurrection, et parce que Valmont et Merteuil, en jouant avec les souvenirs à l'intérieur de ce théâtre, sont aussi dans le ressouvenir. Ce ressouvenir redoublé, ressouvenir qui s'écrit par-dessus le ressouvenir, comme un palimpseste, par la résurrection et par le jeu, est à la fois un espoir de répétition et de nouveauté, pour cela un espoir de dépassement des limites. C'est cela Quartett, une ouverture à l'illimité, à travers une double théâtralisation du ressouvenir. Un présent surdéterminé par la mort et un désir d'avenir. Une quête de vérité sur fond d'explosion. Une explosion qui détruit le « je/corps » et le reconstruit avec ses ruines, sur ses ruines. Une explosion qui détruit les frontières de l'identité, qui déchire la chair, et qui reconstruit à travers le passé et le vide, espace à remplir, l'espoir de l'à-venir, l'espoir du devenir « je/corps », qui se place au-delà de l'humain, au-delà de ce qui est insupportable : la mort, la violence, la cruauté, etc.

Dans son autobiographie, Müller dit ceci au sujet du terrorisme :

Je trouve l'indignation morale contre le terrorisme sans objet, et c'est pour moi une hypocrisie, c'est pour cela que cette phrase centrale de Fatzer est si importante pour moi, le mot humble. Tuer, avec humilité, c'est le ferment théologique du terrorisme. Il n'y a pas de solution, c'est le paradoxe humain. [...] Quartett est une réaction au problème du terrorisme, avec un contenu, avec un matériau, qui superficiellement n'a rien à voir avec cela ${ }^{22}$.

Qu'est-ce que Quartett? "Une réaction au problème du terrorisme » sur le terrain de l'érotisme, un combat à mort où je tue l'autre avec humilité, le jeu cruel du «je suis bourreau » et du «je suis victime », un jeu de masques, jeu de la vérité et du mensonge, qui éclaire l'intimité autant qu'il la détruit. Le jeu de Valmont et Merteuil est comme un barrage à l'angoisse de la mort, et pourtant seule la mort libère de l'angoisse. Paradoxe insupportable que le théatre supporte. Pour reprendre le mot de Müller, Quartett est, superficiellement, l'affirmation du vide qui naît de l'expérience de la finitude de l'être, expérience qui explose les limites de la chair et les frontières du je. Profondément, une interruption du politique, à travers la communauté formée par le désir, par ce qui se joue intimement entre un homme et une femme, à travers le conflit du je et de l'autre, à travers la suspension de l'Histoire et du temps. Cette interruption fait apparaitre la violence potentielle du collectif: comment affronter l'insupportable, sans dieu, sans idéologie, sans mensonge ? Le théâtre est sans aucun doute une réponse, peut-être même la seule. Le théâtre, pour Müller, doit affronter ce que l'existence ne peut pas supporter : l'inhumain. Par nécessité, il doit poser la question fondamentale suivante : Qu'est-ce que l'humain? Pour cela, il faut interroger, observer, affronter, exploser les frontières. Mais l'énigme perpétuelle qu'est la liberté n'est pas loin. 


\section{NOTES}

2. Jean-Pierre Morel, L'hydre et l'ascenseur, essai sur Heiner Müller, Circé, 1996, p. 194.

3. Heiner Müller, Quartett, dans La Mission, trad. J. Jourdheuil et B. Perregaux, Minuit, 1982.

4. Heiner Müller, Guerre sans bataille, Vie sous deux dictatures, L'Arche, 1996, p. 269.

5. Heiner Müller cité dans Günther Heeg, Empire Mort Allemagne Théâtre de la résurrection, article de la revue Théâtre/Public 160-161, numéro consacré à Heiner Müller « Généalogie d'une oeuvre à venir ", 2001, p. 88.

6. Georges Bataille, L'expérience intérieure, Gallimard Tel, 1954, p. 86.

7. Ibid. p. 86.

8. On peut noter ici que dans la mise en scène de Quartett au théâtre de l'Odéon en 2006, par Robert Wilson, Merteuil, interprétée par Isabelle Huppert, répète mécaniquement et comme à l'infini des phrases du monologue, comme pour signifier une solitude éternelle, un noir profond.

9. Roland Barthes, Fragments d'un discours amoureux, Seuil, 1977, p. 126.

10. Ibid., p. 124.

11. Roland Barthes, Écrits sur le théâtre, Seuil, 2002, p. 340.

12. Friedrich Nietzsche, Le Gai savoir, in Euvres T. II, Laffont coll. Bouquins, 1993, p. 211.

13. Georges Bataille, in Euvres complètes VIII, Gallimard, 1976, p. 492.

14. Catherine Naugrette, Paysages dévastés, Circé, 2004, p. 146.

15. Maurice Blanchot, La communauté des amants, in La Communauté inavouable, 1983, Minuit, p. 71.

16. Günther Heeg enseigne les études théâtrales à l'Université de Mayence et à l'Université de Leipzig.

17. Dans la revue Théâtre/Public, op. cit., p. 88.

18. Julia Kristeva, Histoires d'amour, Folio essais, 1983, p. 90.

19. Georges Bataille, L'Érotisme, Minuit, 1957, p. 150.

20. Matthias Langhoff, metteur en scène, proche de Heiner Müller, a mis en scène Quartett en 2006.

21. Sorën Kierkegaard, Le Concept d'angoisse, traduction P. H. Tisseau, Alcan, p. 43.

22. Dans Guerre sans bataille, op. cit., p. 268.

\section{AUTEUR}

\section{LAURE COUILLAUD}

Doctorante à l'Université Paris 7 